\title{
Circles and Sounds
}

\author{
Sarah Linebaugh \\ 790 Huff Road NW, Apt. 5020 \\ Atlanta GA 30318 \\ 281-793-0449 \\ slinebaugh9@gmail.com
}

\begin{abstract}
The exploration of real-time, interactive motion graphics in a live environment is a response to the exponential growth of technology in culture and the human desire to engage in meaningful experiences. Interactive installations draw from the concepts of the New Aesthetic and Relational Aesthetics to create art that depends on cutting-edge technology while emphasizing human interaction and connectedness. The use of graphics that respond in real-time are a hallmark of the medium. Immediate responses from the technology allow the user to engage with the artwork instantaneously. By doing so, they can create meanings that are unique to themselves.
\end{abstract}

My interactive art installation, Circles and Sounds, combines art and technology in a way that will allow the user to engage with the technology to create their own real-time graphics and sound experience that is completely unique to them.

\section{Categories and Subject Descriptors}

J.5 [Computer Applications]: Arts and Humanities - Arts, fine and performing; I.4.8 [Computing Methodologies]: Image Processing and Computer Vision, Scene Analysis -Depth Cues; Tracking; Motion;

\section{Keywords}

Interactive art, Installation, Motion graphics, Real-time, Kinect, Quartz Composer

\section{INTRODUCTION}

In recent history, society has seen two trends develop. The first is the exponential growth of technology and society's increasing dependence on it and interdependence with it. The second has developed as a result of the first: as society is immersed deeper into technology, the desire to step away from the isolation it can bring has become stronger. This is a push toward two seemingly different directions: the desire for the latest and greatest and a craving for meaningful interactions. Out of these desires, new trends are developing in the art world that combine both of them in explorations that are both cutting edge and innately human. Interactive art in live settings often uses technology in ways that have been previously unseen, and it encourages a pioneering spirit in developing new technologies. At the same time, many of these works are inoperable or meaningless without human engagement and creation.
Interactive art references aesthetics like the New Aesthetic ${ }^{1}$ and Relational Aesthetics ${ }^{2}$ to create a combination that is both uniquely technical and collaborative. The New Aesthetic draws from the visual language of pixels, bytes and glitches given to us by the machines we use. It is an aesthetic completely unique to the last few generations, yet it has become so prevalent within our culture that it is being cultivated as style, as art, and as language. Relational Art or Relational Aesthetics is an aesthetic that bases itself on the concept of art as a form of social exchange. The artist is seen as the catalyst in relational art, rather than being at the center. The aesthetic creates inter-subjective encounters in which meaning is established collectively, rather than individually.

Another important aspect of interactive art is that it takes place in real-time. Immediate responses from the technology allow the user to engage with the artwork instantaneously. By doing so, they can create meanings that are unique to themselves. Interactive art is often on the cutting edge of the latest technological developments since many of the artworks are based on new ideas that have not been done before. Because of this, interactive art is intimately connected to the electronics, code, and maker/hacker communities, which is completely alien to other forms of art.

The field of motion graphics has found itself merging into interactive art as well. The use of graphics in a live setting has grown exponentially in recent times. Graphics are no longer confined to televisions and computer screens but are found at concerts, in outdoor projection, on walls, in museums, and many other places. Graphics that respond in real-time are often integral parts of interactive artworks, and many companies that previously created motion graphics for television or film are now using graphics in unique ways that engage with a multitude of environments.

Interactive art, and specifically motion graphics in interactive art, is born out of an effort to respond to two seemingly disparate trends in society. Its use of new, cutting edge technology to connect with the core human desire to create meaningful interactions is something that is new to the art world and is only recently beginning to find its feet. ${ }^{3}$ As technology continues to evolve, interactive art will be at the forefront of creating meaningful connections for its audiences in ways that correspond with their current cultural landscapes. 


\section{THE NEW AESTHETIC AND RELATIONAL AESTHETICS}

\subsection{The New Aesthetic}

The New Aesthetic is an aesthetic that has existed within culture for quite a while but has only recently been given a name. In May of 2011, a young English man named James Bridle, armed with a few photos and a Tumblr blog, announced what some are calling the next big art movement: "The New Aesthetic." Bridle showcased imagery of art made with Google Earth photos, lowpolygon styles in car and shoe design, and pixel patterns in art, design, fashion, landscaping and architecture - all pointing to the idea that the visual language of the machine has been appropriated by humans, and we are using it to express ourselves and our ideas. Engaging the technical as human beings is something that is relatively new to the past few generations, but it is now an unavoidable part of our culture. The New Aesthetic is just the label assigned to the visible manifestations of this new cultural reality.

\subsection{Relational Aesthetics}

A second aesthetic that has been discussed in recent times is that of Relational Aesthetics. Spearheaded by French critic Nicolas Bourriaud, Relational Art or Relational Aesthetics is "a set of artistic practices which take as their theoretical and practical point of departure the whole of human relations and their social context, rather than an independent and private space." 2 In his book, Relational Aesthetics, Bourriaud focuses on art as a form of social exchange. He draws attention to the artist as someone who could be viewed as the catalyst in relational art, rather than being at the center. The concept seeks to establish inter-subjective encounters in which meaning is established collectively rather than individually. In her article, "Antagonism and Relational Aesthetics," Claire Bishop describes art works fitting within this aesthetic as something that is entirely beholden to the contingencies of its environment and audience rather than a discrete, portable, autonomous work of art:

This audience is envisaged as a community: rather than a one-to-one relationship between work of art and viewer, relational art sets up situations in which viewers are not just addressed as a collective, social entity, but are actually given the wherewithal to create a community, however temporary or utopian this may be. ${ }^{4}$

The goal of the aesthetic is to emphasize the temporal, the collective experience, and possibly, the creation of a functioning 'microtopia' in the present. This emphasis on togetherness and the creation of a common experience is central to Relational Aesthetics.

So how do these two aesthetics intersect? Since before Bourriaud first put pen to paper in the nineties to comment on the art world's response to the human desire for "face-to-face" interaction in the wake of the virtual relationships of the Internet, this interplay between humans and machines has been at work. We have seen culture both embrace the machine and all of its pixel-ly aesthetics and language, yet also push back with the desire for more human interaction. However, especially in recent years, there has been an initiative to marry them both. Specifically in the art world, there have been numerous installations that combine both the New Aesthetic and the Relational Art, both the high-tech and the interactive.

\section{INTERACTIVE ART: TECHNOLOGY AND HUMANITY}

\subsection{What is interactive art?}

One trend that has been seen in the art world in the last twenty years is that of interactivity in installation art. Many artists who engage in producing work in this vein rely on science and technology to work with their aesthetic concept in order to make the artwork a reality. In their article, "Living Laboratories: Making and Curating Interactive Art," Lizzie Muller and Ernest Edmonds define interactive art as "audience and machine...working in dialogue to produce a unique artwork for each audience encounter." 5 They refer to Edmond's earlier work, where he describes interactive art as a matrix of the artist, the audience and the artifact, or the "art system." Each element of the matrix is actively involved in the creation of the artwork, and "meaning occurs through the process of exchange, and interactivity itself is the very medium of the work".

Simon Penny, in his article, "From A to D and Back Again: The Emerging Aesthetics of Interactive Art," defines what interactivity means and how it can be applied to artwork:

An interactive system is a machine system which reacts in the moment, by virtue of automated reasoning based on data from its sensory apparatus. An Interactive Artwork is such a system which addresses artistic issues. A painting is an instance of representation. A film is a sequence of representations. Interactive artworks are not instances of representation, they are virtual machines which themselves produce instances of representation based on real-time inputs. ${ }^{6}$

He describes interactive art as having two tasks: first, to discover the nuances and modalities of the interactive dynamic and figure out how to apply them to the aesthetic goals. Second, to integrate the aesthetically manipulated interactive dynamic with the other components of the work. The central aspect to these tasks (and to the artwork that is created from them) is time. Penny stresses the concept of real-time as being the most important aspect of interactive art:

The difference between an interactive application and handwritten correspondence is essentially one of time. There is a time frame which is perceived by humans as 'real-time' response, it is the time frame of physiological reaction. Slower (or faster) than this is not 'real-time'. Switching a light switch produces a real-time response, but it is simplistic. Interactivity allows reactions by a machine in real time which are intellectually and cognitively complex. This defines the medium. ${ }^{6}$

It is this real-time response that makes interactive art extremely engaging and enables it - despite its added trappings of technology - to tap into the distinctly human need for connection 
and experience. In this aspect, interactive art can be seen as a cousin of Relational Art, an aesthetic that engages with concepts of human relations and environments.

\subsection{The maker/hacker movement and open- source technology}

Because real-time response is a crucial aspect of the medium, using technology to achieve this has become an integral part of interactive art. Many interactive installations rely on open-source software and technology and often use techniques or resources that correspond with the renegade maker/hacker mentality. ${ }^{7}$ These resources are typically developed by interested communities, and developers are usually volunteers. Access to source code is open and free, and licenses are non-restrictive. Because these resources are easy to obtain, the communities surrounding them are often robust and provide valuable information and support in their application. This open-source mentality has led to continued exploration and invention with these technologies, which allows for the spread of unique concepts and unlimited possibilities to create something new. This creative usage of technology has found a natural home within interactive installations, where it is used in unique ways to bring the artist's vision to life.

\section{CIRCLES AND SOUNDS INSTALLATION}

Using the principles I have found in my research of interactive art and motion graphics, the Circles and Sounds installation allows the user to create their own graphics and sound experience. Incorporating the principles of the New Aesthetic and Relational Aesthetics, the installation draws from the language of technology while requiring a human element for creation. Graphics and sounds respond in real-time to positioning and gestures by participants, allowing them to facilitate the creation of the artwork.

Drawing from open-source technology and information from the maker/hacker community, a software program called Quartz Composer is used for creation of the visuals. Motion tracking information is provided through use of a Microsoft Kinect. The graphics consist of circles that overlay each other in a particle system. As the user moves around in front of the installation, the circles follow. The height of the circles will be determined by the height of the user's arms. If the arms are raised, the circles will rise. If the arms are lowered, the circles will lower. The size of the circles will be based on the relative position of the user as well. The closer the user gets to the installation, the smaller the circles become. The farther away the user positions him or herself, the larger the circles become.

Sound also corresponds to the movements of the user. Using the Kinect in conjunction with the audio software program Ableton Live, users are able to manipulate the pitch of the track playing by raising or lowering their arms. Moving closer or farther away will manipulate volume.

The installation will consist of a projector and a blank canvas that the graphics will be projected onto. When a person approaches the canvas, the Kinect picks up on their movements and triggers the applications. Quartz Composer is used to read the joint information sent by the Kinect to control the graphics, and Ableton Live is used to control sound. The graphics are pre-made in After Effects.

Through the building and execution of this installation, I hope to explore many of the principles and techniques previously discussed in this thesis, such as the New Aesthetic, Relational Aesthetics, interactivity in art and real-time responsiveness, the use of graphics in a live setting, projection, and the creation of graphics in real-time.
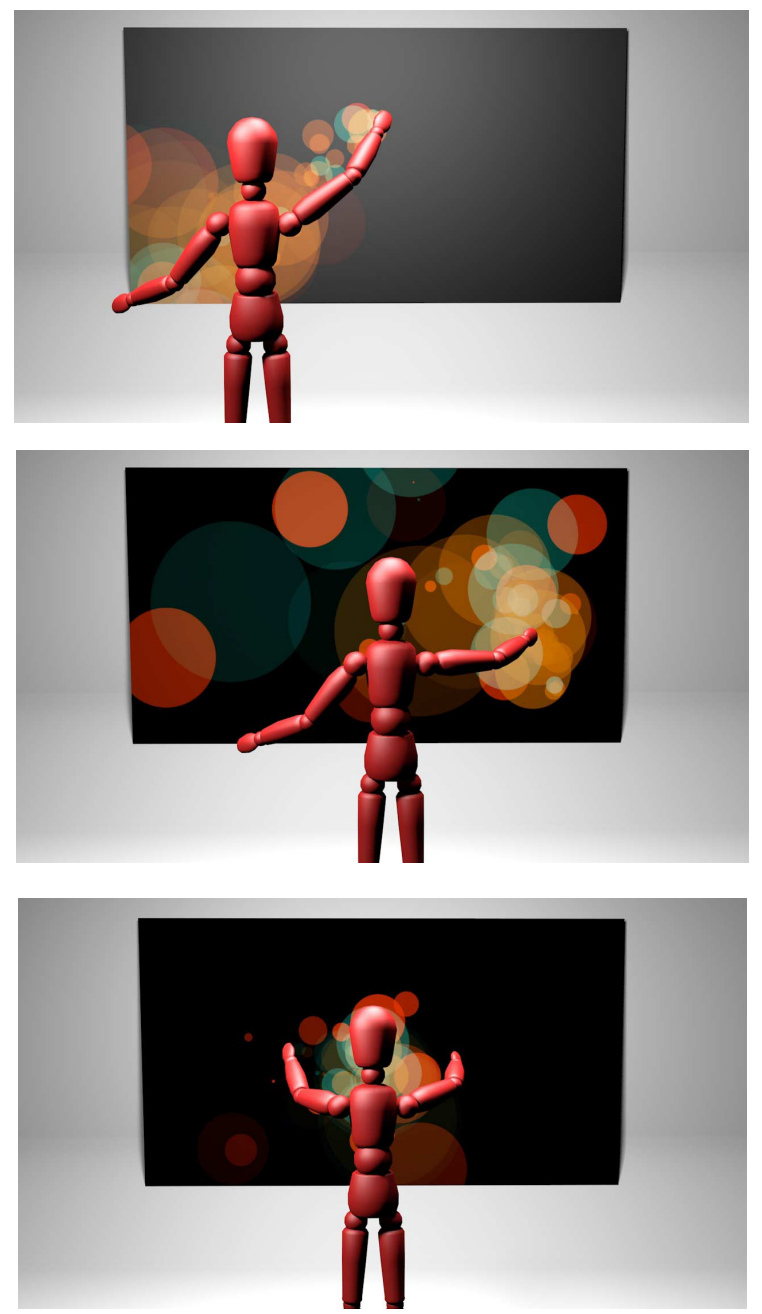

Figure 1. Mock-ups of the installation in progress. As the figure moves his arms and re-positions his body, the circles follow and respond to his gestures.

Technology has become an entrenched part of our society, often pushing people away from connectedness and social exchange, but through this project I will explore how it can be used to bring people together and facilitate human interaction. I also hope to explore the concept of the "artist" and the creation of art as a collective experience. The project will also examine art as a temporal event. Unlike a sculpture or a painting, this piece will only exist for a certain period of time and will be dependent upon those who chose to participate. In that sense, it differs greatly from "works of art" in the traditional sense, which are made by one particular person and exist indefinitely. 


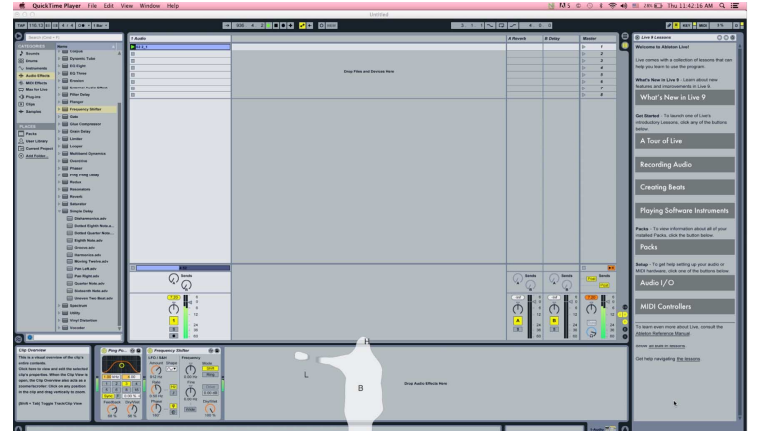

Figure 2. Ableton Live corresponding with data from the Kinect. As the figure moves his arms and re-positions his body, the sounds will react and respond in accordance.

\section{CONCLUSION}

Real-time, interactive installations are positioned in a unique place. They have feet firmly planted in both the art world and the science world. They have a deep dependency on technology because they require real-time responsiveness, but they also require human input to even be brought into existence. This combination allows for the fusing of the New Aesthetic and Relational Aesthetics to perhaps bring about a completely different aesthetic that has yet to be defined.

Interactive art has also expanded the field of motion graphics. Graphics that respond in real-time are often integral parts of interactive artworks, and motion design has found a home in a variety of live settings, such as projections, concerts, events, museums, and galleries. Interactive art also challenges the concept of the "artist." Many of the pieces require active participation from their admirers rather than passive observation. As such, they are created collectively rather than by a single person. The "artist" can be seen more as a facilitator than the sole creator.

Interactive art, and specifically motion graphics in interactive art, allows technology to connect with the core human desire to create meaningful interactions. Its potential is limited only by the ideas behind the works themselves. As technology continues to evolve, interactive art will continue to be at the forefront of creating significant experiences for its audiences and allow them to use technology in ways that bring them closer together.

\section{REFERENCES}

1. Sterling, Bruce. "An Essay on the New Aesthetic." Wired. 2 April 1996. Web. 11 March 2013

2. Bourriaud, Nicolas. Relational Aesthetics. Les presses du reel, 2002. Print.

3. Holt-Lunstad, Julianne, Timothy B. Smith, J. Bradley Layton. "Social Relationships and Mortality Risk: A Metaanalytic Review.” PLos Medicine, 7.7. July 2010. Web. 11 July 2014.

4. Bishop, Claire. "Antagonism and Relational Aesthetics." George Baker. 110 (2004): 49-50. Web. 5 Feb. 2013.

5. Muller, Lizzie and Ernest Edmonds. "Living Laboratories: Making and Curating Interactive Art." Electronic Art and Animation Catalog. (2006): 147-149. Web. 11 March 2013.

6. Penny, Simon. "From A to D and Back Again: The Emerging Aesthetics of Interactive Art." Leonardo Electronic Almanac. April 1996. Web. 11 March 2013.

7. Chayka, Kyle. "How Open Source is Disrupting Visual Art." The Creator's Project. 4 April 2012. Web. 11 July 2014. 